\title{
A chromatin target for ALL
}

\section{By Chris Cain, Associate Editor}

\begin{abstract}
A New York University School of Medicine-GlaxoSmithKline plc collaboration has shown that an inhibitor of the chromatin regulator JMJD3 could help treat T cell acute lymphoblastic leukemia. ${ }^{1}$ As JMJD3 is upregulated and used as a notch partner in T cell leukemia specifically, and not during T cell develop-
\end{abstract} ment, the researchers hope that targeting the epigenetic modulator could avoid the safety pitfalls of general notch pathway suppression.

Current treatments for $\mathrm{T}$ cell acute lymphoblastic leukemia (T-ALL) involve combination chemotherapy regimens that have considerable toxic side effects. Apart from drugs that inhibit BCR-ABL tyrosine kinase fusion proteins, no targeted therapies are marketed for the disease.

The notch pathway is particularly attractive for drug development because it is overactivated in the majority of T-ALLs. However, most attempts have fallen short because notch signaling is involved in many other cellular processes, and it has been difficult to find selective inhibitors that do not have severe safety liabilities.

"Current notch-targeted therapies are pan-notch inhibitors and are burdened with side effects that limit their applicability," said Patrick Trojer, head of biology at epigenetics company Constellation Pharmaceuticals Inc.

At least one notch 1 (NOTCH1)-targeted molecule, OncoMed Pharmaceuticals Inc.'s anti-NOTCH1 antibody, OMP-52M51, is in Phase I trials for solid tumors and lymphoid malignancies. The program is part of an OncoMed-GSK collaboration.

In 2012, a group led by Panagiotis Ntziachristos, a postdoctoral fellow in the laboratory of NYU School of Medicine professor Iannis Aifantis, identified chromatin regulators that facilitated notch signaling in T-ALL. ${ }^{2}$ That study found that up to $25 \%$ of T-ALL cases had inactivating mutations in proteins that form the polycomb repressive complex 2 (PRC2). It also showed that loss of trimethylated histone H3 lysine 27 (H3K27me3) occurs commonly in T-ALL. PRC2 trimethylates $\mathrm{H} 3 \mathrm{~K} 27$ and can silence gene expression.

Because that work did not yield an obvious drug target, Ntziachristos looked for enzymes that might be targeted for drug development. The logic was that because H3K27me3 was downregulated in T-ALL, inhibiting enzymes that demethylate $\mathrm{H} 3 \mathrm{~K} 27 \mathrm{me} 3$ could provide therapeutic handles.

The project narrowed in on two highly homologous enzymes that catalyze demethylation of H3K27me3: JMJD3 (histone demethylase jumonji domain containing 3; KDM6B) and lysine-specific demethylase 6A (KDM6A; UTX).

"We pursued JMJD3 and UTX for two reasons," Ntziachristos said. "First, because of the importance of lysine 27 trimethylation in the oncogenic expression and transcription of notch targets, and second, because we were trying to find new therapeutic avenues for this disease." However, to the team's surprise, the two enzymes showed opposite effects. Whereas JMJD3 appeared to stimulate NOTCH1mediated cell growth, UTX was a tumor suppressor.

In xenograft mouse models of T-ALL, JMJD3 shRNA decreased cell proliferation compared with control shRNA. By contrast, UTX shRNA increased proliferation compared with control. In addition, studies in samples from patients with T-ALL found UTX-inactivating mutations associated with the disease. This suggested that inhibiting JMJD3 could help treat T-ALL but inhibiting UTX could be detrimental.

The key question was what would happen if both targets were simultaneously inhibited because the close homology of the two enzymes would make it difficult to design a selective inhibitor. The group got lucky when a team from the Structural Genomics Consortium (SGC) and GSK published a series of nanomolar-potent inhibitors of JMJD3 and UTX. "It was at this point a small miracle occurred," Ntziachristos told SciBX. "We had completed all of the genetic studies and molecular characterization of these targets, and it was incredibly fortuitous timing when GSK and the SGC published their description of a JMJD3 and UTX inhibitor."

Aifantis and Ntziachristos began collaborating with Laurens Kruidenier and Rab Prinjha, head of GSK's EpiNova Epigenetics Discovery Performance Unit. GSK provided the academics with an active inhibitor and an inactive control along with advice on how best to use them. In a panel of human T-ALL cell lines and patient samples, the dual JMJD3-UTX inhibitor decreased cell growth compared with the inactive control. Thus, the authors concluded that the effect of inhibiting JMJD3 was dominant over the effect of inhibiting UTX enzymatic activity. Kruidenier is a biology discovery leader at GSK.

Prinjha told SciBX, "While molecular techniques like knockouts or knockdowns can show some advances, you often need a small molecule to push them forward, and that was the case here, a real example of chemical biology."

Results were published in Nature.

\section{Safety notch}

Although JMJD3 inhibitors might provide a safer way to tamp down notch signaling than directly targeting NOTCH1, at this point that is 


\section{ANALYSIS}

still just theory because off-target effects have not been studied. To get evidence in vivo, the compounds will need to be optimized for oral availability.

Ntziachristos believes the approach has a potential safety advantage because JMJD3 does not undergo the same expression changes as members of the notch pathway, and it does not seem to be used by the pathway during development and in cellular processes outside of oncogenesis.

"The need for H3K27 demethylation may be much higher and more urgent in an oncogenic context. Notch levels increase during $\mathrm{T}$ cell development and in other contexts, whereas JMJD3 levels generally remain stable, and JMJD3 knockout cells can have normal T cell development and differentiation," he said.

Trojer agreed that targeting the KDM6 family members JMJD3 and UTX could provide a safety advantage. "KDM6 inhibitors may be a lesstoxic alternative, especially if it turns out that KDM6 interacts specifically with NOTCH1 but not with other notch family members. However, KDM6 inhibitors need to be further improved before potential safety issues could be explored."

According to Prinjha, the findings also strengthen the safety case for developing a dual JMJD3-UTX inhibitor. "If you look at the molecular data in the literature, you would have shied away from hitting UTX at all-you would have been scared off by mutations implicated in cancer. In this context, the activity of the small molecule provides some interesting opportunities, and from a safety perspective I'm now a lot more comfortable with a joint UTX and JMJD3 inhibitor."

Kristian Helin, CSO of EpiTherapeutics ApS, was cautious about making predictions about the safety of the approach and emphasized that a bioavailable inhibitor must be tested in animal models before any conclusions could be drawn. He added that he would like to see such compounds tested as part of combination regimens with other T-ALL therapies.
EpiTherapeutics is developing histone demethylase inhibitors and expects to advance a compound against an undisclosed target into the clinic in 2015. Helin said the company has considered JMJD3 but is prioritizing other targets. Helin is also director of the Biotech Research \& Innovation Centre at the University of Copenhagen.

GSK is continuing to collaborate with the NYU School of Medicine team but declined to disclose next steps. The patent and licensing status of the work is undisclosed.

Cain, C. SciBX 7(35); doi:10.1038/scibx.2014.1033

Published online Sept. 11, 2014

\section{REFERENCES}

1. Ntziachristos, P. et al. Nature; published online Aug. 17, 2014; doi:10.1038/nature13605

Contact: lannis Aifantis, New York University School of Medicine, New York, N.Y.

e-mail: iannis.aifantis@nyumc.org

Contact: Aristotelis Tsirigos, same affiliation as above e-mail: aristotelis.tsirigos@nyumc.org

Contact: Charles G. Mullighan, St. Jude Children's Research Hospital, Memphis, Tenn.

e-mail: charles.mullighan@stjude.org

2. Ntziachristos, P. et al. Nat. Med. 18, 298-303 (2012)

3. Kruidenier, L. et al. Nature 488, 404-408 (2012)

\section{COMPANIES AND INSTITUTIONS MENTIONED}

Constellation Pharmaceuticals Inc., Cambridge, Mass. EpiTherapeutics ApS, Copenhagen, Denmark GlaxoSmithKline plc (LSE:GSK; NYSE:GSK), London, U.K. New York University School of Medicine, New York, N.Y. OncoMed Pharmaceuticals Inc. (NASDAQ:OMED), Redwood City, Calif.

Structural Genomics Consortium, Oxford, U.K. University of Copenhagen, Copenhagen, Denmark 